\title{
Imaging community-acquired pneumonia in children
}

\author{
Savvas Andronikou ${ }^{1,2,3}$
}

Received: 27 March 2017 / Accepted: 6 April 2017 / Published online: 20 September 2017

(C) Springer-Verlag Berlin Heidelberg 2017

Community-acquired pneumonia in children is common in all parts of the world and it remains the major single cause of death in children outside the neonatal period. The presentation and natural progression of pneumonia is constantly changing with the use of new vaccines, and clinicians must adapt to these changes. This issue's minisymposium on imaging of pneumonia starts with a review by Le Roux and Zar [1], who report, for example, that there have been more empyemas since the introduction of pneumococcal conjugate vaccine. This change in disease profile might change the likelihood of a clinician requesting an imaging study. On the other hand, the choice of which imaging modality to use is also evolving with advances in technology and with emerging knowledge regarding diagnostic imaging. Both clinicians and radiologists are involved in making the diagnosis of pneumonia, and these groups need clarity on their respective roles. Mahommed and colleagues [2] describe one environment where clinicians and radiologists are engaging in discussions through the World Health Organization Chest Radiography in Epidemiological Studies project and where definitions of chest radiograph findings representing pneumonia are being revised for vaccine trials. This process has come about because of criticism and dissatisfaction with the previous radiographic World Health

Savvas Andronikou

docsav@mweb.co.za

1 Department of Paediatric Radiology, Bristol Royal Hospital for Children, Upper Maudlin Street, Bristol BS2 8BJ, UK

2 Department of Paediatric Radiology, University of Bristol, Bristol, UK

3 Department of Radiology, University of Cape Town, Cape Town, South Africa
Organization definitions for pneumonia. The World Health Organization has grappled with definitions that now include some size specifications but has continued to embrace chest radiography as the primary diagnostic imaging tool for confirming the presence of pneumonia.

Despite the numerous clinical guidelines advising against performing any imaging in children with suspected uncomplicated community-acquired pneumonia, Andronikou et al. [3] report on how clinicians continue to request chest radiographs to make the initial diagnosis of pneumonia, in both developing and developed regions of the world. In the developing world setting, one underlying rationale for requesting radiographs in suspected community-acquired pneumonia is to exclude tuberculosis as a possible cause for the symptoms. In this regard, the poor accuracy and reliability of chest radiographs for the diagnosis of both pneumonia and tuberculosis are highlighted in the review.

Having set the scene regarding the evolving clinical environment, the continued use of chest radiographs by vaccine trial groups and clinicians who defy the guidelines and the problems with chest radiographs, the three papers that follow describe the cross-sectional imaging techniques for diagnosing pneumonia and its complications. Stadler and colleagues [4] lead the way by describing the use of US for the detection of consolidation to diagnose pneumonia. They summarise the many recent publications authored predominantly by clinicians, of which the majority fail to test US against a true reference standard. This major weakness of the research to date plays into the hands of denialists and contributes to paediatric radiologists' slow support for US in the diagnosis of childhood pneumonia. If one assumes that imaging is needed for the diagnosis and management of paediatric communityacquired pneumonia, then US would present a desirable option in that it can be used as a point-of-care test in emergency departments and outpatient rooms and it can be performed 
safely by clinicians. The published results show it to be more reliable than chest radiography for the diagnosis of consolidation. On the other end of the diagnostic imaging spectrum, Liszewski and colleagues [5] report on the recent advances in MRI, which have resulted in faster and shorter protocols, making it feasible for diagnosing pneumonia and its complications. However, in the real world the modality is not yet widely distributed enough or affordable enough to represent a global first-line tool. It is more likely that this technique will continue to represent a radiation-free problem-solving alternative in the immediate future. Most current protocols still require children to lie still for many minutes at a time, and young children are often anaesthetised for the duration of a study. Anaesthesia is considered by many to be a risk to the child and must be weighed against risks of other diagnostic imaging options. The finale of this minisymposium by Andronikou and colleagues [6] re-examines the gold standard for imaging the lungs - CT. Despite its superiority in imaging the lung parenchyma, airways, mediastinum, pleural space and thoracic cage, CT has taken a hit from the poor perceptions about radiation dose to children. Despite this, advances such as iterative reconstruction now make it possible for low-dose CT scans to be performed in the radiation range equivalent to about 10 radiographs. Doses in this range must be viewed sensibly according to new information on radiation-induced deoxyribonucleic acid (DNA) damage and repair. In this review, we note these improvements in CT dose in addition to highlighting other advantages of $\mathrm{CT}$ such as its speed, its accuracy in identifying pneumonia, its suitability for demonstrating the complications of pneumonia and its utility in exposing lymphadenopathy, which is a key factor in the diagnosis of tuberculosis [6]. For CT to be considered a first-line tool in the imaging of suspected community-acquired pneumonia, the reduced radiation doses must be appreciated and publicised by the wider radiologic fraternity in the context of the minor potential future risks, while the significant limitations of conventional chest radiographs for diagnosing pneumonia and its complications should be acknowledged.

This minisymposium on the imaging of childhood pneumonia presents modern issues facing the paediatric radiologist and the referring clinician. The authors, who are experts in their field, represent experience from both developed and developing countries and therefore provide a global outlook. The overarching message is that radiologists' imaging decisions and techniques must be adapted to the ever-changing face of pneumonia that has resulted from ongoing vaccination efforts; they must respond to the evolving requirements of our clinical colleagues; and they must reflect the improving capabilities of new imaging technology and new knowledge that emerges. It should also be remembered that guidelines and imaging protocols do not fit every scenario. The developing world presents the radiologist with different challenges and additional potential diagnoses that require alternative thinking strategies. I hope you enjoy the read.

\section{Compliance with ethical standards}

Conflicts of interest None

\section{References}

1. Le Roux DM, Zar HJ (2017) Community-acquired pneumonia in children - a changing spectrum of disease. Pediatr Radiol. doi:10. 1007/s00247-017-3827-8

2. Mahomed N, Fancourt N, de Campo J et al (2017) Preliminary report from the World Health Organisation Chest Radiography in Epidemiological Studies project. Pediatr Radiol. doi:10. 1007/s00247-017-3834-9

3. Andronikou S, Lambert E, Halton J et al (2017) Guidelines for the use of chest radiographs in community-acquired pneumonia in children and adolescents. Pediatr Radiol. doi:10.1007/s00247-0173944-4

4. Stadler JAM, Andronikou S, Zar HJ (2017) Lung ultrasound for the diagnosis of community-acquired pneumonia in children. Pediatr Radiol. doi:10.1007/s00247-017-3910-1

5. Liszewski MC, Görkem S, Sodhi KS et al (2017) Lung magnetic resonance imaging for pneumonia in children. Pediatr Radiol. doi:10. 1007/s00247-017-3865-2

6. Andronikou S, Goussard P, Sorantin E (2017) Computed tomography in children with community-acquired pneumonia. Pediatr Radiol. doi:10.1007/s00247-017-3891-0 